\title{
Implant Site Development by Orthodontic Forced Eruption of Nontreatable Teeth: A Case Report
}

\author{
Amir Reza Rokn ${ }^{1}$, Anna Saffarpour ${ }^{2}{ }^{2}$, Rouzbeh Sadrimanesh ${ }^{3}$, Kaveh Iranparvar ${ }^{4}$, Aida Saffarpour ${ }^{5}$, \\ Majid Mahmoudzadeh ${ }^{6}$ and Ahmad Soolari ${ }^{7}$
}

\author{
${ }^{I}$ Dental Implant Research Centre, Tehran University of Medical Sciences, Iran \\ ${ }^{2}$ Department of Periodontology, Tehran University of Medical Sciences International Campus, Iran \\ ${ }^{3}$ Prosthodontist, Private Practice, Tehran, Iran \\ ${ }^{4}$ Endodontist, Private Practice, Tehran, Iran \\ ${ }^{5}$ Department of Restorative Dentistry, Hamedan Dental University, Iran \\ ${ }^{6}$ Department of Orthodontics, Hamedan Dental University, Iran \\ ${ }^{7}$ American Board of Periodontology; Private Practice, Silver Spring, MD, USA
}

\begin{abstract}
Background: Loss of bone and soft tissue attachment are common sequelae of periodontitis that may jeopardize the aesthetic outcome and compromise the functional and aesthetic outcomes of treatment. The following case report describes one of the most predictable techniques of vertical ridge augmentation, which is orthodontic extrusion or forced eruption of hopeless teeth.

Method: A 34-year-old woman who presented with severe attachment loss and deep pockets was diagnosed with generalized aggressive periodontitis. The mobile maxillary incisors were consequently extracted and were replaced with dental implants. However, prior to extraction, orthodontic extrusion of the hopeless incisors was performed to correct vertical ridge defects. Following extrusion and extraction of the maxillary incisors, to prevent soft tissue collapse and to preserve the papillae during socket healing, the crowns of the extracted teeth were used as pontics on a removable partial provisional denture. After 8 weeks, the implants were placed, and an immediate functional restoration was delivered. After 4 months of healing, a fixed definitive partial prosthesis was fabricated and delivered.
\end{abstract}

Result: After periodontal treatment, over a 2-year period, the progression of aggressive periodontitis was controlled. The mean vertical movement of marginal bone was $3.6 \mathrm{~mm}$. The use of the crowns of extracted teeth appears to be an effective method to maintain papillae.

Conclusions: Orthodontic extrusion is a predictable method for the correction of vertical ridge defects. Orthodontic treatment does not aggravate or hasten the progression of aggressive periodontitis.

Keywords: Bone augmentation, dental implants, dental papilla, forced eruption, orthodontic extrusion, vertical ridge augmentation.

\section{INTRODUCTION}

Bone and attachment loss are consequences of periodontitis and can compromise the functional and aesthetic outcomes of periodontal treatment [1]. To overcome this problem, predictable therapeutic methods are needed to rebuild the lost tissues. Horizontal ridge augmentation is one of the conventional and relatively predictable methods; however, vertical ridge augmentation (VRA) may not be as predictable. The most important disadvantages of the techniques

*Address correspondence to this author at the Department of Periodontology, Tehran University of Medical Sciences International Campus, Iran, Tel: 0098-9122025016; Fax: 00982188375546;

E-mail: anna_1359_sa@yahoo.com used for VRA, such as guided bone regeneration, sinus elevation, and distraction osteogenesis, are not only that they are invasive, but also that the outcomes of such treatments are not predictable [1].

Forced eruption or orthodontic extrusion of hopeless teeth is a nonsurgical, noninvasive technique by which the volume of residual bone at the future implant sites can be increased [2]. Forced eruption or orthodontic extrusion of hopeless teeth was introduced in 1993 by Salama and Salama [3]. When a tensile force is applied to the periodontal ligament, the fibers are stretched, and the osteoblasts produce new bone $[4,5]$.

During extrusion, not only the crestal bone but also the supporting soft tissues move vertically. Moreover, Kajiyama et al. showed that, during orthodontic extrusion, the free gin- 
giva moved about $90 \%$ and the attached gingiva about $80 \%$ of the extruded distance [6]. Forced eruption of the teeth not only increases the dimensions of the alveolar bone [7, 8], but it also moves the free gingiva and interdental papillae coronally, whereas the location of the mucogingival junction remains constant. This can enhance aesthetics, especially in the anterior maxilla. For that reason, studies and case reports have reported the use of forced eruption for the management of interdental papillae before placement of implants [9].

In the present case report, aesthetics and function were re-established in a patient who suffered from generalized aggressive periodontitis (GAP) via forced eruption, papilla preservation, and implant placement.

\section{CASE DESCRIPTION AND RESULTS}

A 34-year-old white woman was referred to the Department of Periodontics at Tehran Medical University in 2006. Her chief complaints were mobility and spacing between her maxillary teeth that had developed during the past year, gingival bleeding, and swelling. The patient was especially concerned about all four maxillary incisors (Fig. 1).

She had a history of periodontal disease and had undergone periodontal surgery three times, at the ages of 14, 22, and 27 years, in the posterior regions of both arches. All previous treatments had been performed by a general dentist. The medical history of the patient's family revealed that her mother had lost her teeth in her youth because of loosening; her brother had also suffered from severe periodontal disease.

All the teeth were present except for the mandibular right third molar. The patient had poor oral hygiene, with a Plaque Index of 97\%, and bleeding on probing (BOP) was observed in all regions. Radiographic evaluations revealed moderate to severe generalized bone loss (Fig. 1). The mobility was grade I at teeth \#5, \#14, \#15, \#26, and \#30; grade II at teeth $\# 1, \# 2, \# 3, \# 4, \# 7, \# 10, \# 12, \# 13, \# 23$, \#24, and \#25; and grade III at teeth \#8 and \#9. In addition, tooth \#3 had grade I distal furcation involvement, and tooth \#30 had grade I buccal furcation involvement. According to the clinical and radiographic findings, the patient was diagnosed with GAP. The objectives of treatment were to control the advancement of the disease, reduce tooth mobility, re-establish aesthetics and function, and, in general, obtain predictable and sustainable results.

Treatment began with instruction in oral hygiene. Next, scaling and root planing were performed. Amoxicillin (250 $\mathrm{mg}$ ) and metronidazole $(250 \mathrm{mg})$ three times a day and $0.2 \%$ chlorhexidine mouth rinse (two times a day) were prescribed for 7 days afterward.

Since poor oral hygiene had caused recurrence of the disease, the patient entered a maintenance phase before initiating any further treatment. After 6 months, the Plaque Index had decreased to $16 \%$, but BOP was still about $40 \%$. Mobility of the teeth had decreased, except in the anterior maxilla. In addition, although a decrease in pocket depth (average of $2 \mathrm{~mm}$ ) was observed, pockets deeper than $5 \mathrm{~mm}$ and BOP were still present in all regions.
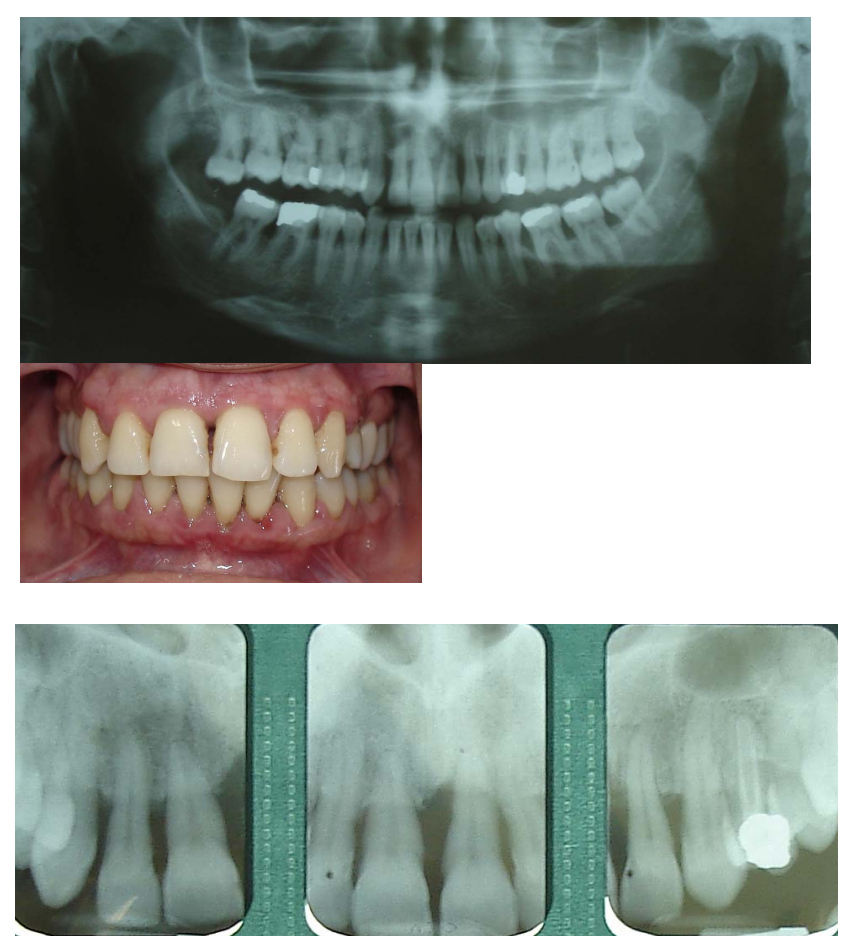

Fig. (1). Above: Initial panoramic and clinical views of occlusion and tooth alignment. Below: Periapical radiographs of the maxillary incisors.

During phase II of treatment, to eliminate the deep pockets, periodontal flaps and correction of bone contours (osteoplasty) were performed in all but the anterior maxilla. No significant decrease in the mobility of the maxillary incisors was observed. Grade III mobility of the central incisors made mastication very difficult. The crown/root ratio of the central incisors was about $2 / 1$, which explained the severe mobility (Fig. 1c). Because of these problems and the unpleasant appearance of the central incisors, we decided to extract them. The patient insisted on replacement of her lost teeth without any damage to adjacent teeth.

In agreement with the patient's wishes, and taking into consideration the crown/root ratio of the canines and premolars and the extent of the future edentulous area (Fig. 1b), the decision was made to place implants. However, pronounced, severe bone loss had occurred in the anterior maxilla as a result of the GAP, such that the extractions might lead to collapse of the soft tissue, aggravation of vertical defects of the ridge, and vertical bone resorption. For these reasons, forced eruption of the hopeless teeth was used to decrease the amount of bone resorption.

Tomography and standard periapical radiography were used to evaluate the extruded bone and assess alterations in the crestal bone. Before orthodontic treatment, the distance between the floor of the nose and the marginal bone of each hopeless tooth was evaluated and registered. These distances ranged between 12.8 and $15.7 \mathrm{~mm}$ for the maxillary incisors (Fig. 2). Edgewise brackets were bonded to the surfaces of all maxillary teeth between and including the first molars (Fig. 3). 


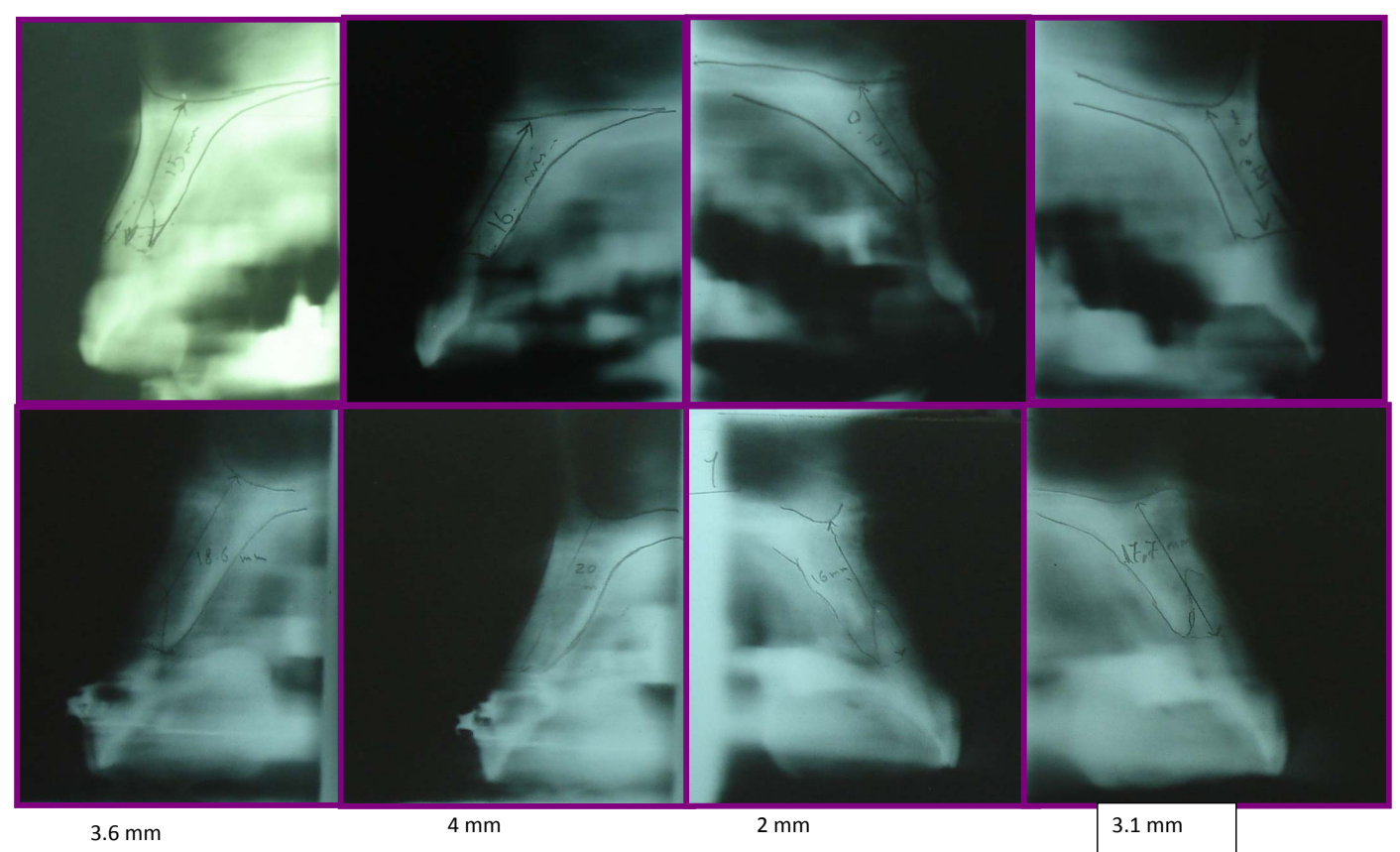

Fig. (2). Tomographic scans of the maxillary incisors before treatment (top row) and after treatment (bottom row). Left to right: right lateral incisor, right central incisor, left central incisor, left lateral incisor.

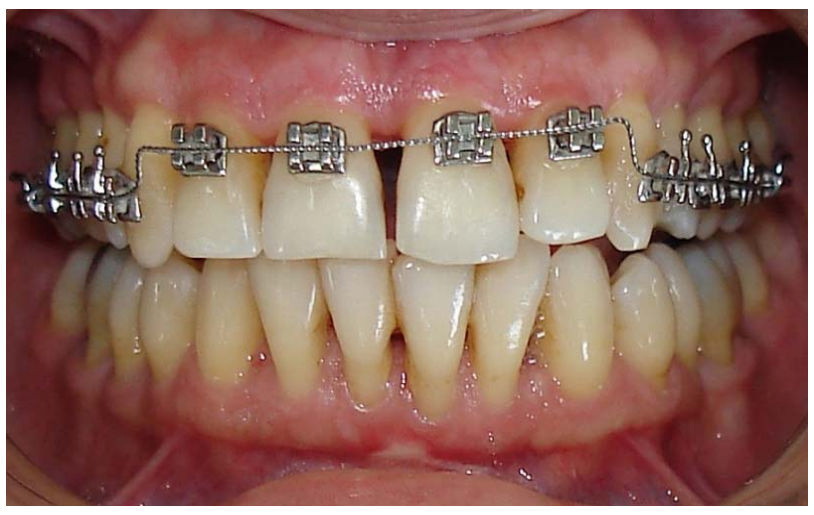

Fig. (3). Forced eruption was initiated.

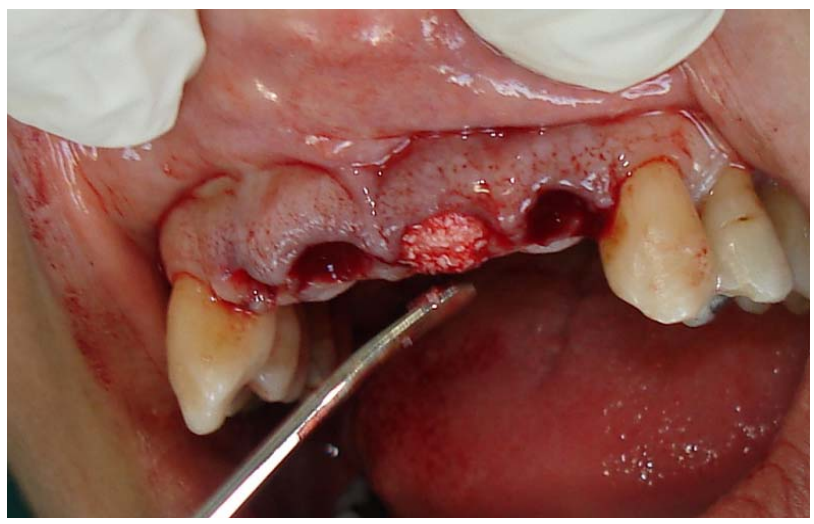

Fig. (4). After extraction, the central incisor sockets were filled with Bio-Oss up to the level of the alveolar crest.

Extrusion was performed over the course of 4 months. The patient was checked every 2 weeks, and all interferences between the palatal surfaces of the anterior maxillary teeth and the incisal edges of the anterior mandibular teeth were eliminated. When the original and final casts (after 16 weeks) were compared, the teeth were determined to have been displaced $4 \mathrm{~mm}$ from their baseline locations.

In extrusion of the hopeless teeth, the bone level of the adjacent teeth dictates the amount of extrusion. Comparison of the marginal bone levels of the lateral incisors with those of the canines revealed that the amount of extrusion was sufficient after 4 months. To compensate for the bone resorption that would inevitably follow implant placement, the orthodontic extrusion was allowed to progress somewhat beyond the marginal bone levels of the canines (slight overtreatment).

Comparison of radiographs obtained before and after extrusion revealed that the relationship between the cementoenamel junction and the marginal bone remained constant. In addition, the depth of the vertical defect at the mesial of the left central incisor had decreased.

After 4 months of stabilization, the maxillary incisors were extracted. Before extraction of the teeth, standardized tomography was performed using a template. The amount of bone that had been regenerated in the incisor area ranged between 2 and $3.9 \mathrm{~mm}$ (Fig. 2). The incisors were extracted gently. The sockets of the central incisors were filled with bone grafting material (Bio-Oss) up to the level of the crestal bone (Fig. 4).

Extraction of the teeth without preservation of the soft tissue will lead to soft tissue collapse and loss of the interdental papillae, and the resultant dark spaces in the interproximal regions will compromise the aesthetic results of treatment. Therefore, to preserve the soft tissue architecture and ensure a better aesthetic result, the extracted teeth were used as pontics during healing of the extraction sites. The extracted teeth were cut $2 \mathrm{~mm}$ below the gingival zenith, pulpal tissues were removed, and the cut surfaces were shaped into ovate pontics using composite resin. After the crowns were placed into their respective sockets, they were 

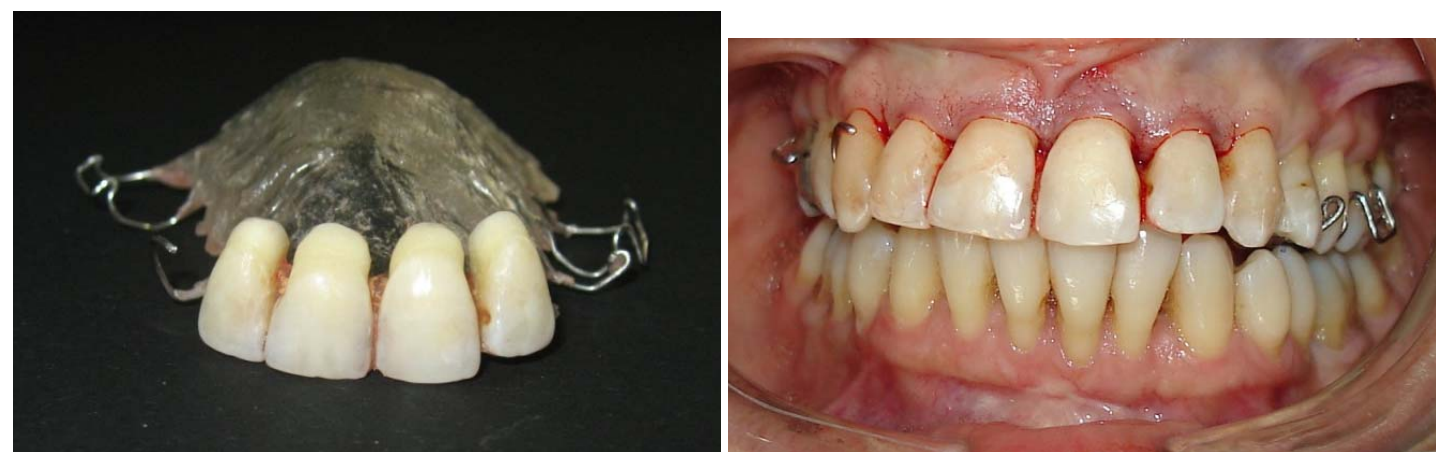

Fig. (5). Left: The remodeled extracted teeth were attached to a previously fabricated removable acrylic resin partial denture in their original positions. Right: Provisional denture in use.

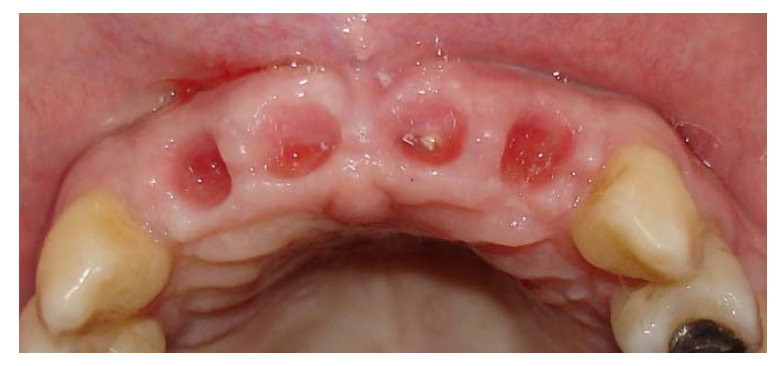

Fig. (6a). View of the sockets at 5 weeks after extraction.

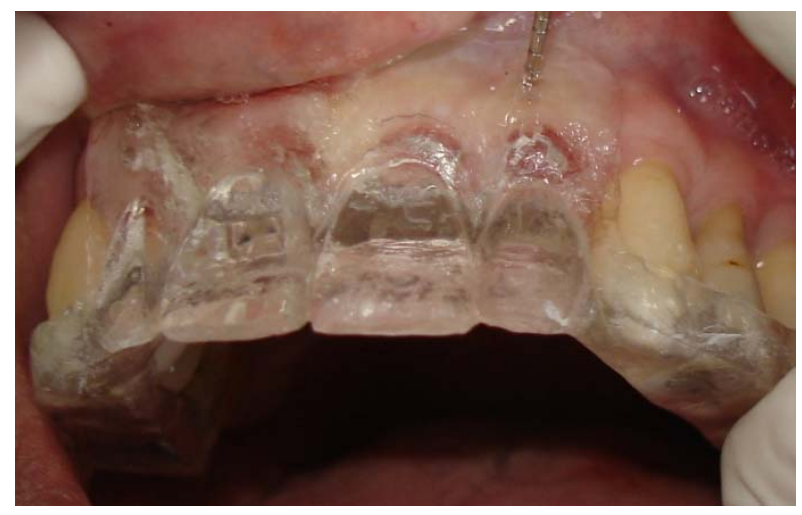

Fig. (6b). Bone mapping using an acrylic resin template.

attached to each other using flowable composite. The positions of the teeth were registered using a silicon index. The reshaped extracted teeth were attached to a previously fabricated removable acrylic resin partial denture in their original positions (Fig. 5a), and the denture was delivered to the patient about 3 hours after extraction of the teeth. The patient was instructed to wear the prosthesis continuously except for cleaning. This continuity of use was essential to maintain soft tissue form (Fig. 5b).

To keep the Bio-Oss granules in place, the patient was advised not to remove her denture earlier than 1 week after surgery to perform oral hygiene. Amoxicillin $250 \mathrm{mg}$ and Metronidazole $250 \mathrm{mg}$ (every 8 hours for 7 days) were prescribed. No abnormal clinical signs or symptoms of inflammation were seen during the first week.

One month after extraction of the teeth, epithelialization of the socket surfaces was complete (Fig. 6a). During the healing period, minor soft tissue recession of about $1 \mathrm{~mm}$ was observed at the gingival margin and interdental papillae.

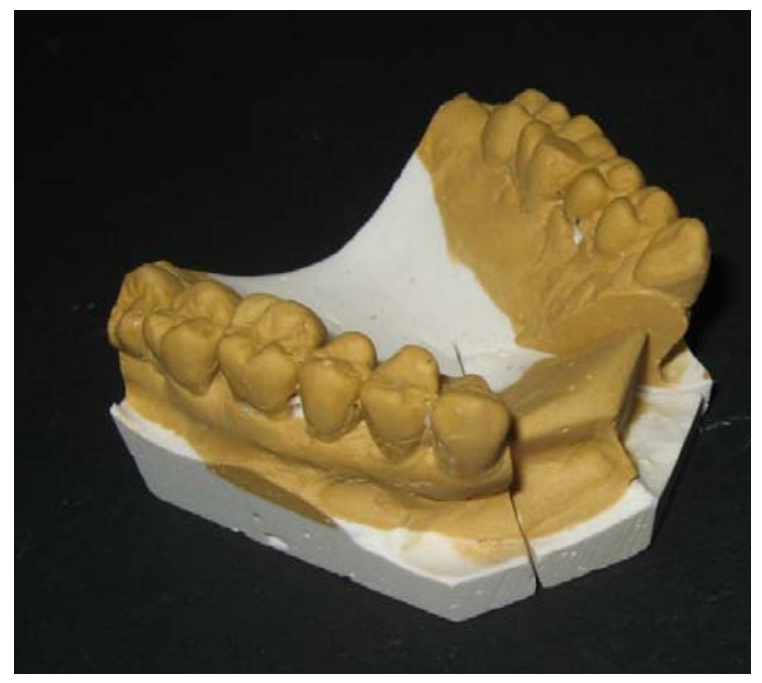

Fig. (6c). Shaping of the cast, based on the results of bone mapping.

Because raising flaps in the edentulous region for the placement of implants might increase the risk of tissue recession, a model-based, flapless implant placement technique was used. For this reason and to evaluate the bone volume after extraction of the teeth and determine the ideal paths of implants, a template was made using transparent acrylic resin (Fig. 6b). Four holes were made at the buccal and lingual surfaces of the template at the lateral incisor sites. After local anesthesia had been administered, bone mapping was achieved via the holes made on the template. The template was inserted back onto the cast, and the stone was reshaped based on the recorded measurements. The outcome of the reshaping thus accurately represented the patient's residual bone (Fig. 6c). Model surgery was done, and implant analogues were inserted into the cast to determine the primary paths of the implants. Access holes to the implant analogues were prepared in the template and were filled with guttapercha. After a cone beam computed tomogram was obtained with the template in place, the final paths of the implants were corrected on the template (Fig. 6d).

On the day of surgery, two implants, each $3.5 \mathrm{~mm}$ wide and $15 \mathrm{~mm}$ long, were placed in the lateral incisor sites using the template with the flapless method. Immediately after surgery, an implant-level impression was taken. Within 2 hours, a provisional prosthesis had been prepared and was delivered to the patient without any functional occlusion. 


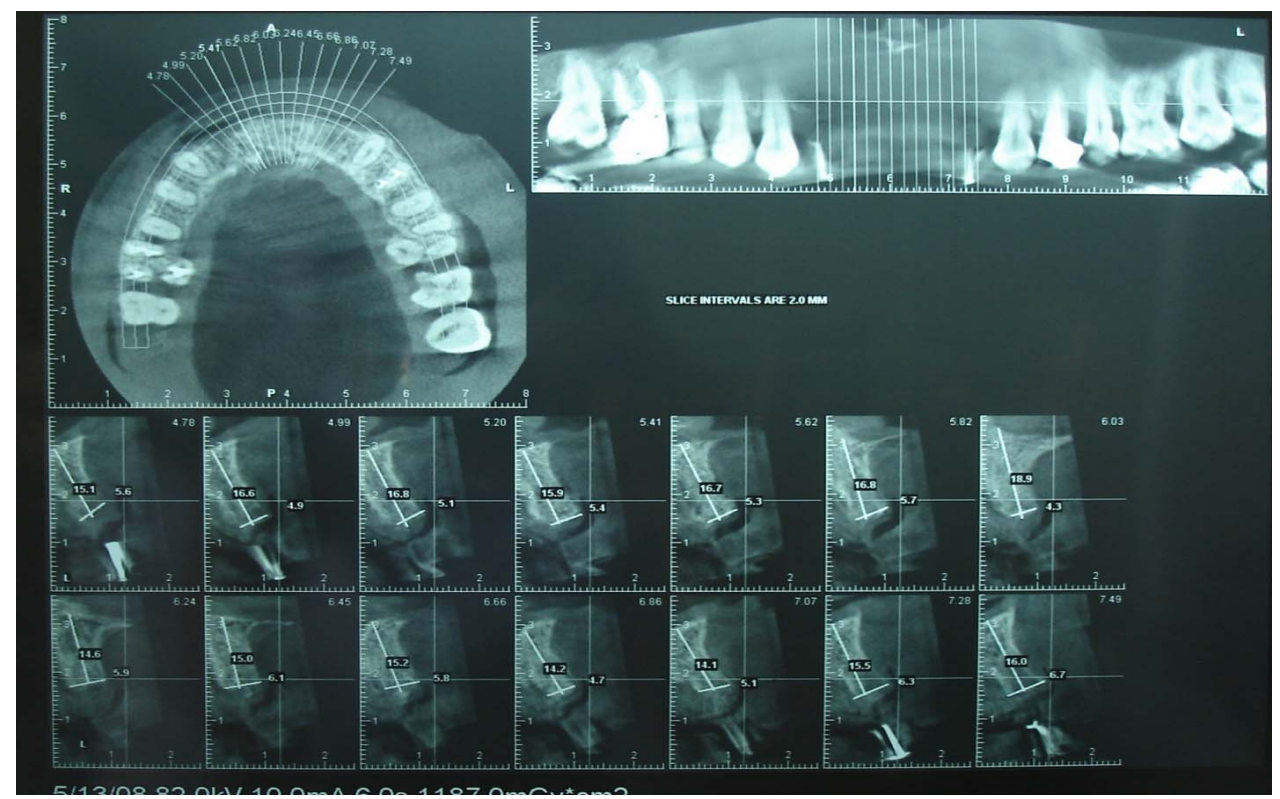

Fig. (6d). Cone beam computed tomography with the template in place.

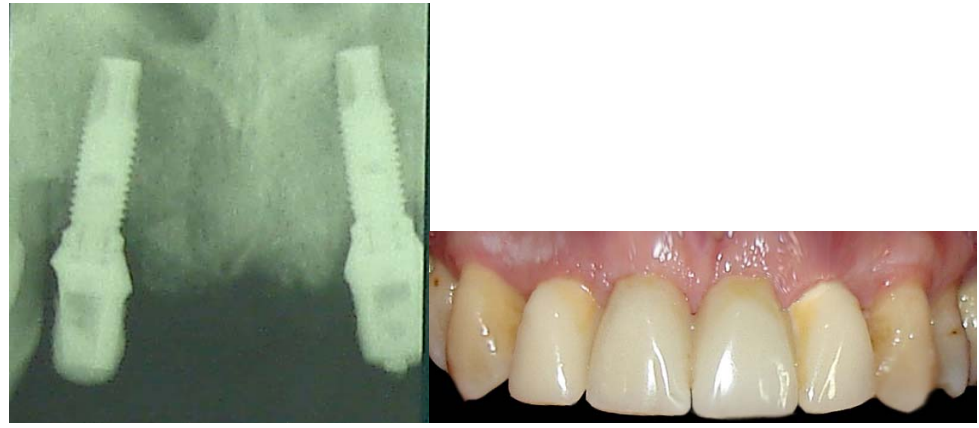

Fig. (7). Left: Periapical radiograph at 4 months after implant placement. Right: The definitive restoration.

The patient was instructed to maintain oral hygiene and to avoid biting with the anterior teeth. After 1 week, the sutures were removed. The definitive prosthesis was delivered to the patient after 4 months (Fig. 7).

\section{DISCUSSION}

In the present patient, GAP was controlled with conventional periodontal treatments, and, over the course of 3 years of follow-up treatment, continued maintenance stopped the progression of the disease. In this patient, severe recession, mobility, and migration were observed in the anterior maxilla. The treatment plan employed correction of the hard tissue dimensions through forced eruption, soft tissue management via the preparation of ovate pontics using the crowns of the extracted teeth, and, finally, placement of implants with a flapless technique.

The use of orthodontic techniques for ridge augmentation before placement of implants has been poorly documented to date, and most studies that have investigated this possibility are case reports [9-11]. In 1992, Salama and Salama introduced the technique of extruding hopeless teeth with the aim of improving the hard tissue dimensions at implant placement sites. They called their technique orthodontic extrusive remodeling. In this technique, after extrusion of the hopeless teeth and a period of stabilization, the hopeless teeth are extracted and implants are placed at the sites [3]. With this nonsurgical technique, which employed orthodontic principles, a three-dimensional improvement in the hard and soft alveolar tissues was obtained, so that placement of prosthetically oriented implants was possible. This is particularly important from the viewpoint of aesthetics, especially in the anterior maxilla. Mantzikos and Shamus later used this technique to improve the dimensions of the anterior maxillary ridge before placement of implants. They showed that forced eruption was an efficient means of accomplishing this goal and also provided desirable aesthetic results in the definitive restoration [11].

During extrusive movements, the depth of pockets related to any teeth affected with periodontal disease will decrease. Mantzikos and Shamus explained this process as "reversion or pocket lining" [10]. However, the application of a tipping force in the facial direction during forced eruption can lead to an increase in the faciolingual dimension of the ridge $[12,13]$.

In the present case, the crowns of extracted anterior maxillary teeth were cut $2 \mathrm{~mm}$ apical to the cementoenamel junction and were formed into ovate pontics. The ovate pontics were employed to help preserve (1) the clot on the graft materials and tooth socket, (2) the interdental papillae and gingival contours, and (3) the emergence profile. Because of the extent of the edentulous region in anterior maxilla, it was not 
possible to connect the pontics to the adjacent teeth with composite or fiber-reinforced composite; for this reason, the crowns were attached to a removable partial denture.

During the healing process, the surface of the sockets was lined with epithelium, and no clinical inflammation was observed in the tissues around the pontics. After 1 month, the color of this epithelium was normal; only the centers of sockets in direct contact with the pontics were red.

This clinical report has described the restoration of nontreatable teeth in the aesthetic zone using forced orthodontic eruption to develop stable peri-implant soft tissue that is in aesthetic harmony with the surrounding tissues. Orthodontic forced extrusion achieved a coronal shift of bone and gingiva and enhanced the recipient implant site without extensive surgical ridge augmentation procedure. The non-surgical management reported in this case is appealing to most patients. The results of this technique in this case are in agreement with a report by Brindis and Block [14], who stated that an intact sulcular attachment apparatus is necessary for coronal movement of soft tissues and that this is dependent on bone formation, since the tooth is extruded on the vector of the movement of the tooth and the rate of tooth extrusion is affected by the degree of attachment between the alveolar bone and the tooth.

Our patient lost her maxillary anterior teeth early in life, when she was 34 years old. However, her periodontal disease had begun much earlier. Susceptibility to periodontal disease at an early age is not uncommon. Early onset periodontal disease often presents as a "silent disease" that affects millions of children and young adults, and the high prevalence of periodontitis in adults is often related to undiagnosed early onset periodontal disease [15].

\section{CONFLICT OF INTEREST}

None declared.

\section{ACKNOWLEDGEMENT}

None declared.

\section{REFERENCES}

[1] McAllister BS, Haghighat K. Bone augmentation techniques. J Periodontol 2007; 78: 377-96.

[2] Korayem M, Flores-Mir C, Nassar U, Olfert K. Implant site development by orthodontic extrusion: a systematic review. Angle Orthod 2008; 78: 752-60.

[3] Salama H, Salama M. The role of orthodontics extrusive remodeling in the enhancements of the soft and hard tissue profiles prior to implant placement: a systematic approach to the management of extraction site defects. Int J Periodontics Restorative Dent 1993; 13: 312-33.

[4] Reitan K. Clinical and histologic observations on tooth movement during and after orthodontic treatment. Am J Orthod 1967; 53: 72145 .

[5] Reitan K. Tissue behavior during orthodontic tooth movement. Am J Orthod 1960; 46: 881-900.

[6] Kajiyama K, Murakami T, Yokota S. Gingival reactions after experimentally induced extrusion of the upper incisors in monkeys. Am J Orthod Dentofacial Orthop 1993; 104: 36-47.

[7] Ingber JS. Forced eruption, Part I: a method of treating isolated one and two wall infrabony defects: rationale and case report. J Periodontol 1974; 45: 199-206.

[8] Ingber JS. Forced eruption, Part II: a method of treating nonrestorable teeth: periodontal and restorative considerations. J Periodonto 1976; 47: 203-16.

[9] Lin CD, Chang SS, Liou CS, Dong DR, Fu E. Management of interdental papillae loss with forced eruption, immediate implantation, and root-form pontic. J Periodontol 2006; 77: 135-41.

[10] Mantzikos T, Shamus I. Forced eruption and implant site development: soft tissue response. Am J Othod Dentofacial Orthop 1997; 112: 596-606.

[11] Mantzikos T, Shamus I. Forced eruption and implant site development: case report. Angle Orthod 1998; 68: 179-85.

[12] Zuccati G, Bocchieri A. Implant site development by orthodontic extrusion of teeth with poor prognosis. J Clin Orthod 2003; 37: 307-11.

[13] Nozawa T, Sugiyama T, Yamaguchi S, et al. Buccal and coronal augmentation using forced eruption and buccal root torque: a case report. Int J Periodontics Restorative Dent 2003; 23: 585-91.

[14] Brindis MA, Block MS. Orthodontic tooth extrusion to enhance soft tissue implant esthetics. J Oral Maxillofac Surg 2009; 67(11 suppl): 49-59.

[15] Nowzari H, Botero JE. Latin america: native populations affected by early onset periodontal disease. J Calif Dent Assoc 2011; 39(6): 383-91.

Received: February 12, 2012

Revised: March 08, 2012

Accepted: March 08, 2012

(C) Rokn et al.; Licensee Bentham Open.

This is an open access article licensed under the terms of the Creative Commons Attribution Non-Commercial License (http://creativecommons.org/licenses/by-nc/3.0/) which permits unrestricted, non-commercial use, distribution and reproduction in any medium, provided the work is properly cited. 